\title{
L'exposition professionnelle aux produits cancérogènes dans les Pays de la Loire
}

\author{
Occupational exposure to carcinogens in the Pays de la Loire \\ region
}

A. Grellier ${ }^{a *}$, J.-F. Certin ${ }^{b}$, J.-Y. Dubréc, Y. Roquelaure ${ }^{a, d}$

a Consultation de pathologie professionnelle, CHU d'Angers, 4, rue Larrey, 49933 Angers cedex

9. France

${ }^{\mathrm{C}}$ CRAM des Pays de la Loire, 2, place de Bretagne, 44932 Nantes cedex 9, France

${ }^{\mathrm{C}}$ DRTEFP Pays de la Loire, 26, boulevard Vincent-Gâche, BP 46339, 44263 Nantes cedex 2, France

'Laboratoire d'ergonomie et d'épidémiologie en santé au travail, CHU d'Angers, 4, rue Larrey, $\checkmark 49933$ Angers cedex 9, France

\author{
Disponible en ligne sur \\ $\because$ ScienceDirect \\ www.sciencedirect.com
}

\section{Résumé}

Objectif. Cibler les populations salariées les plus exposées aux cancérogènes dans les Pays de la Loire, caractériser l'exposition professionnelle à ces produits et la comparer à l'exposition nationale. Méthode. Exploitation de l'enquête SUMER 2003. Réalisation d'un calage à la marge de l'échantillon qui permet l'extrapolation des résultats obtenus sur l'échantillon à la population régionale. Comparaison des résultats régionaux aux résultats nationaux.

Résultats et discussion. Les hommes sont davantage exposés aux cancérogènes que les femmes. Des différences sociales majeures apparaissent en termes d'exposition aux produits cancérogènes : $25,0 \%$ des ouvriers sont exposés à des produits cancérogènes, alors que seuls $4,4 \%$ des salariés des autres catégories socioprofessionnelles le sont. Les moins de 30 ans sont plus exposés que les autres. Parmi les ouvriers exposés, $45 \%$ sont exposés à au moins un produit cancérogène avec une intensité élevée. Dans ces situations rencontrées principalement dans la construction et l'industrie manufacturière, les protections collectives ne sont présentes que dans un tiers des situations d'exposition et les protections individuelles sont trop peu souvent mises à disposition. L'enquête SUMER permet de pointer les difficultés de mise en place de mesures préventives pour lutter contre l'exposition aux produits cancérogènes (lorsque la substitution n'est pas possible) et d'identifier les populations à cibler en priorité pour améliorer cette prévention : les jeunes ouvriers. (C) 2009 Elsevier Masson SAS. Tous droits réservés.

\footnotetext{
* Auteur correspondant.

e-mail : amelie.ducept@cegetel.net
}

1775-8785X/\$ - see front matter @ $\odot 2009$ Elsevier Masson SAS. Tous droits réservés. 


\section{Introduction}

Le cancer est devenu, en 2004, la cause de décès la plus fréquente en France devant les maladies cardiovasculaires [1]. II constitue la première grande cause de mortalité prématurée (40\% des décès survenus avant 65 ans en Pays de la Loire) [2]. Les facteurs de risque de cancer sont divers et peuvent se cumuler. On estime que $4 \%$ de l'ensemble des cancers sont attribuables à des facteurs professionnels [3,4]. II est très probable que ce taux soit sous-estimé et qu'il puisse atteindre 15 à $20 \%$ pour certains cancers tels que le cancer bronchopulmonaire. Selon l'InVS, entre 11000 et 23 ooo nouveaux cas de cancer d'origine professionnelle apparaîtraient chaque année en France [5]. Réduire l'exposition aux cancérogènes en milieu de travail est une priorité pour lutter contre le cancer. Pour cela, il faut notamment systématiser la surveillance épidémiologique des personnes exposées à des risques cancérogènes sur leur lieu de travail [6]. L'enquête Surveillance médicale des risques professionnels (SUMER) 2003 a permis d'évaluer à 2370000 le nombre de salariés exposés à des produits cancérogènes en France, soit 13,5\% des salariés du champ de l'enquête [7]. La catégorie socioprofessionnelle la plus touchée est celle des ouvriers. Dans les Pays de la Loire, la population active est sociodémographiquement différente de la population active française. L'exposition aux produits cancérogènes est donc probablement différente de celle de la France entière. Évaluer le nombre de salariés exposés à des produits cancérogènes dans notre région et caractériser cette population semble donc important. II faut cibler les populations les plus exposées aux produits cancérogènes et déterminer leur niveau d'exposition. Cela permettra non seulement de définir des priorités d'actions de santé au travail concernant l'exposition aux cancérogènes dans les Pays de la Loire, mais également de réaliser à long terme un suivi de l'évolution de cette exposition et des moyens mis en œuvre pour la diminuer dans notre région.

\section{Méthodes}

Nous avons évalué et caractérisé l'exposition aux produits cancérogènes dans les Pays de la Loire à partir des données de l'enquête SUMER 2003. L'enquête SUMER est une enquête nationale transversale périodique menée conjointement par la Direction de l'animation de la recherche, des études et des statistiques (DARES) et la Direction des relations de travail (inspection médicale du travail), en collaboration avec un réseau de médecins inspecteurs régionaux du travail et de médecins du travail volontaires. Son principal objectif est de dresser une cartographie des expositions professionnelles pour la population salariée française $[8,9]$. Les salariés enquêtés sont des salariés du régime général, du régime agricole, des hôpitaux publics, de la poste, de la société nationale des chemins de fer (SNCF) et d'électricité de France/gaz de France
(EDF/GDF), et certains salariés d'Air France. À l'exception des intérimaires qui restent dans le champ quel que soit le type de visite, les salariés inclus sont les salariés vus en visite systématique ou en visite d'embauche différée. Ils sont tirés au sort par le médecin enquêteur. Pour chaque salarié tiré au sort, le médecin du travail doit remplir un questionnaire. Le questionnaire est renseigné de manière anonyme.

La participation des médecins du travail des Pays de la Loire a été particulièrement élevée (plus d'un sur trois contre 20,1\% au niveau national). Sur les 49984 questionnaires de l'enquête SUMER 2002 à 2003, 4027 questionnaires concernent des salariés des Pays de la Loire, ce qui permet une extrapolation de l'échantillon régional [10]. Les questionnaires correspondant aux salariés d'EDF/GDF, de la MSA (agriculture, sylviculture et pêche) ainsi que de la SNCF ont été exclus du fait du faible nombre de questionnaires (respectivement 39, 26 et 24 enquêtés répondants) et donc de l'impossibilité d'interpréter les résultats au niveau régional. Dans les Pays de la Loire, aucun salarié de la poste et d'Air France n'a été enquêté. Le champ étudié se compose donc des salariés du régime général, des hôpitaux publics, des intérimaires et de la MSA hors " agriculture, sylviculture et pêche ". Au total, 3938 questionnaires (97,7 \%) ont pu être exploités.

Comme pour l'exploitation nationale de l'enquête SUMER, un calage à la marge de l'échantillon régional a été réalisé par D. Waltisperger (DARES). Cette méthode permet d'extrapoler les résultats observés sur l'échantillon à la population ligérienne. L'échantillon a été subdivisé en trois morceaux comprenant respectivement : les salariés du régime général et les salariés de la mutualité sociale agricole (MSA) travaillant hors " agriculture, sylviculture et pêche ", les salariés suivis par les médecins du travail des hôpitaux publics et les intérimaires. La pondération a été réalisée à l'aide de la macro CALMAR (calage sur marges) selon plusieurs critères : sexe, groupe d'âge, catégorie socioprofessionnelle, secteurs d'activités regroupés, taille de l'établissement.

Les populations de référence sont issues des Déclarations annuelles de données sociales (DADS) [11]. Chaque morceau a fait l'objet d'une extrapolation particulière. La population ligérienne extrapolée par le calage à la marge de l'échantillon est de 916361 personnes. La pondération des données nationales a été réalisée également à l'aide de la macro CALMAR. Les populations de référence étaient issues des DADS, de l'enquête Emploi, des sources communiquées par les grandes entreprises et de la MSA. Les résultats présentés sont les résultats pondérés. Les fréquences régionales et les fréquences nationales obtenues avant pondération ont été comparées en limitant le champ national au champ retenu pour les données régionales (régime général, hôpitaux publics, intérimaires, MSA hors " agriculture, sylviculture et pêche "), avec un seuil de significativité de $5 \%$. L'analyse des données a été réalisée à partir du logiciel SPSS 15.0. Les résultats ne sont pas présentés lorsque les effectifs bruts sont inférieurs à dix. 
Tableau I

Les produits cancérogènes : taux d'exposition régionale et nationale.

\begin{tabular}{|c|c|c|c|c|c|c|c|}
\hline UE & CIRC & TRG & TRA & Produit & $\begin{array}{l}\text { Nombre de salariés } \\
\text { exposés dans les } \\
\text { Pays de la Loire }\end{array}$ & $\begin{array}{l}\text { Taux d'exposition } \\
\text { régionale pour } \\
1000 \text { salariés }\end{array}$ & $\begin{array}{l}\text { Taux d'exposition } \\
\text { nationale pour } \\
1000 \text { salariés }\end{array}$ \\
\hline- & 1 & 36 Bis & - & Huiles entières minérales & 36946 & 40 & 38 \\
\hline- & $2 A$ & - & - & Gaz d'échappement diesel* & 28060 & 31 & 39 \\
\hline 1 & 1 & 47B & $36 C$ & Poussières de bois*** & 26498 & 29 & 22 \\
\hline- & 1 & $25 \mathrm{~A}$ & - & Silice cristalline & 17027 & 19 & 15 \\
\hline 1 & 1 & 10 Ter & - & Chrome et dérivés & 8680 & 9 & 6 \\
\hline 2 & $2 \mathrm{~A}$ & - & - & Trichloroéthylène & 7796 & 9 & 9 \\
\hline 1 & 1 & 16 Bis & 35 Bis & Goudrons de houille et dérivés & 7593 & 8 & 7 \\
\hline 1 & 1 & 37 Ter & - & Nickel et dérivés & 6594 & 7 & 6 \\
\hline 3 & 1 & - & - & Formaldéhyde* & 5139 & 6 & 9 \\
\hline- & 1 & - & - & $\begin{array}{l}\text { Fumées dégagées par les } \\
\text { procédés dans la métallurgie }\end{array}$ & 4515 & 5 & 6 \\
\hline 2 & $2 B$ & - & - & Fibres céramiques & 4192 & 5 & 6 \\
\hline 1 & 1 & $30 / 30$ Bis & 47/47 Bis & Amiante & 3566 & 4 & 6 \\
\hline 2 & $2 \mathrm{~B}$ & 70 Ter & - & Cobalt et dérivés & 3456 & 4 & 3 \\
\hline 3 & 1 & - & - & Résines formophénoliques & 3315 & 4 & 2 \\
\hline 2 & $2 \mathrm{~B}$ & - & - & $\begin{array}{l}\text { Hydrocarbures aromatiques } \\
\text { halogénés et/ou nitrés }\end{array}$ & 3291 & 4 & 6 \\
\hline- & $2 \mathrm{~A}$ & - & - & Carbures métalliques frittés & 3159 & 3 & 2 \\
\hline- & 1 & & & Fumées de vulcanisation & 2812 & 3 & 2 \\
\hline 1 & 1 & 4 & 19 & Benzène (sauf carburants) & 2449 & 3 & 3 \\
\hline 2 & 1 & $61 \mathrm{Bis}$ & - & Cadmium et dérivés & 1390 & 2 & 2 \\
\hline 1 et 2 & 1 à 3 & 15 Ter & - & Amines aromatiques* & 1359 & 1 & 4 \\
\hline 3 & $2 \mathrm{~A}$ & - & - & Perchloroéthylène* & 1080 & 1 & 3 \\
\hline 2 & $2 \mathrm{~A}$ & - & - & Acrylamide & 725 & 1 & 2 \\
\hline 2 & 1 & - & - & Oxyde d'éthylène & 565 & 1 & 1 \\
\hline- & $2 \mathrm{~A}$ & - & - & $\mathrm{PCB}$ et $\mathrm{PBB}$ & 501 & 1 & 0 \\
\hline 2 & $2 \mathrm{~A}$ & - & - & $\begin{array}{l}\text { Nitrosamines (sauf fumées } \\
\text { de vulcanisation) }\end{array}$ & 295 & o & 1 \\
\hline 2 & $2 \mathrm{~A}$ & - & - & Épichlorhydrine** & 236 & o & 1 \\
\hline 1 & 1 & 20 & 10 & Arsenic et dérivés & 108 & o & 1 \\
\hline- & 1 à 3 & - & - & Cytostatiques* & 609 & 1 & 4 \\
\hline
\end{tabular}

UE : union européenne; CIRC : centre international de recherche sur le cancer; TRG : tableau de maladie professionnellle du régime général; TRA : tableau de maladie professionnelle du régime agricole ; ${ }^{*}: p \leq 5 \% ;{ }^{* *}: p \leq 6 \% ;{ }^{* * *}: p \leq 8 \%$; exposition des salariés du régime général, de la MSA (hors agriculture, pêche, sylviculture), des hôpitaux publics et des intérimaires.

Les quatre principaux cancérogènes auxquels sont exposés les salariés figurent en gras dans le tableau.

Les produits cancérogènes ont été définis à partir des premiers travaux de la DARES et de l'inspection médicale du travail sur l'enquête SUMER 2003. Ainsi, 28 produits, ou familles de produits chimiques, ont été retenus comme cancérogènes parmi les 83 produits, ou familles de produits chimiques, répertoriés dans le questionnaire SUMER 2002 à 2003. Cette identification repose sur l'appartenance aux catégories 1 ou $2 \mathrm{~A}$ du Centre international de recherche sur le cancer (CIRC), ou aux catégories 1 ou 2 de la classification européenne. Les rayonnements ionisants n'ont pas été pris en compte dans le cadre de cette étude. La liste des intitulés retenus figure dans le tableau I.

\section{Résultats}

\section{Description de la population salariée ligérienne}

Le tableau // résume les caractéristiques socioprofessionnelles des populations enquêtées. La population ligérienne extra- polée est de 916361 personnes avec 57,6 \% d'hommes. La répartition selon le sexe de la population nationale est statistiquement identique. L'âge moyen de la population ligérienne est significativement inférieur à celui de la population nationale : 38,0 ans ( $\pm 10,8$ ans) avec un âge minimum de 16 ans et un âge maximum de 64 ans, contre 38,6 ans ( $\pm 10,7$ ans) pour la population nationale extrapolée. Dans les Pays de la Loire, le secteur ouvrier est plus important qu'au niveau national $(43,9 \%$ contre $34,1 \% ; p<0,001)$. Les intérimaires sont également plus représentés. Notre région est aussi une région plus industrielle. Les industries manufacturières et les industries agroalimentaires emploient plus de salariés que dans toute la France (respectivement 29,1 et 6,2 \% de salariés versus 22,3 et $3,5 \%$ pour la France ; $p<0,001$ ).

\section{Population exposée aux cancérogènes}

Parmi les salariés du champ étudiés, 13,4 \% (IC95 \% = 13,4 ; 13,5), soit 123213 salariés ligériens, sont exposés à un ou 
Tableau II

Caractéristiques sociales et professionnelles des populations salariées ${ }^{a}$ françaises et ligériennes enquêtées.

\begin{tabular}{|c|c|c|c|c|c|}
\hline & \multicolumn{2}{|l|}{ France } & \multicolumn{2}{|c|}{ Pays de la Loire } & \multirow[t]{2}{*}{$p$} \\
\hline & Effectifs & $\%$ & Effectifs & $\%$ & \\
\hline \multicolumn{6}{|l|}{ Sexe } \\
\hline Homme & 9315079 & 56,5 & 528096 & 57,6 & ns \\
\hline Femme & 7181817 & 43,5 & 388265 & 42,4 & ns \\
\hline \multicolumn{6}{|l|}{ Taille établissement par tranche } \\
\hline 1 à 9 salariés & 4092111 & 24,8 & 216839 & 23,7 & ns \\
\hline 10 à 49 salariés & 4311842 & 26,1 & 240506 & 26,2 & ns \\
\hline 50 à 199 salariés & 3605505 & 21,9 & 215717 & 23,5 & ns \\
\hline 200 à 499 salariés & 1973077 & 12,0 & 120341 & 13,1 & ns \\
\hline 500 salariés et plus & 2514361 & 15,2 & 122958 & 13,4 & ns \\
\hline \multicolumn{6}{|l|}{ Intérim } \\
\hline Non & 15999850 & 97,0 & 877728 & 95,8 & 0,000 \\
\hline Oui & 497046 & 3,0 & 38633 & 4,2 & 0,000 \\
\hline \multicolumn{6}{|l|}{ Âge décennal } \\
\hline Moins de 30 ans & 3929492 & 23,8 & 238914 & 26,1 & 0,000 \\
\hline 30 à 39 ans & 4939498 & 29,9 & 267016 & 29,1 & 0,007 \\
\hline 40 à 49 ans & 4343436 & 26,3 & 241502 & 26,4 & ns \\
\hline 50 à 59 ans & 3136566 & 19,0 & 166279 & 18,1 & 0,050 \\
\hline 60 ans ou plus & 147904 & 0,9 & 2650 & 0,3 & 0,000 \\
\hline \multicolumn{6}{|l|}{ CSP } \\
\hline Cadres et professions intellectuelles supérieures & 2355152 & 14,3 & 80320 & 8,8 & 0,000 \\
\hline Professions intermédiaires & 3932877 & 23,8 & 199898 & 21,8 & 0,001 \\
\hline Employés administratifs (public/privé) & 2122373 & 12,9 & 99497 & 10,9 & ns \\
\hline Employés de service & 2456440 & 14,9 & 134122 & 14,6 & ns \\
\hline Ouvriers qualifiés & 3628420 & 22,0 & 249632 & 27,2 & 0,000 \\
\hline Ouvriers non qualifiés et agricoles & 2001633 & 12,1 & 152892 & 16,7 & 0,000 \\
\hline \multicolumn{6}{|l|}{$\mathrm{NAFG}_{4}$} \\
\hline Industrie & 3781176 & 22,9 & 269212 & 29,4 & 0,000 \\
\hline Construction & 1191871 & 7,2 & 76280 & 8,3 & 0,026 \\
\hline Tertiaire & 11523849 & 69,9 & 570869 & 62,3 & 0,000 \\
\hline \multicolumn{6}{|l|}{ NAFG17 } \\
\hline Industries extractives & 24768 & 15,01 & 1057 & 0,12 & ns \\
\hline Industries manufacturières & 3680462 & 22,31 & 266330 & 29,06 & 0,000 \\
\hline Production et distribution d'électricité, de gaz et d'eau & 75946 & 0,46 & 1825 & 0,20 & ns \\
\hline Construction & 1191871 & 7,22 & 76280 & 8,32 & 0,026 \\
\hline Commerce, réparations auto/articles domestiques & 2853016 & 17,29 & 148526 & 16,21 & ns \\
\hline Hôtels et restaurants & 746494 & 4,53 & 32189 & 3,51 & ns \\
\hline Transports et communications & 858186 & 5,20 & 44585 & 4,87 & ns \\
\hline Activités financières & 671741 & 4,07 & 31645 & 3,45 & ns \\
\hline Immobilier, location, services aux entreprises & 2958961 & 17,94 & 136915 & 14,94 & ns \\
\hline Administration publique & 292893 & 1,78 & 11502 & 1,26 & 0,013 \\
\hline Éducation & 265784 & 1,61 & 23211 & 2,53 & 0,007 \\
\hline Santé et action sociale & 2028636 & 12,30 & 104913 & 11,45 & 0,000 \\
\hline Services collectifs, sociaux et personnels & 821418 & 4,98 & 36477 & 3,98 & ns \\
\hline Activités des ménages & 22198 & 0,13 & 906 & 0,10 & ns \\
\hline Activités extraterritoriales & 4521 & 0,03 & & & \\
\hline
\end{tabular}

p : significativité fixée à 0,$05 ; n s$ : différence non significative; CSP : catégorie socioprofessionnelle; NAFG4 : nomenclature d'activités française 2003 en quatre postes; NAFG17 : nomenclature d'activités française 2003 en 17 postes.

${ }^{a}$ Salariés du régime général, de la MSA (hors agriculture, pêche, sylviculture), des hôpitaux publics et des intérimaires.

plusieurs produits cancérogènes classés catégorie 1 ou 2 par I'Union européenne (UE) ou 1 ou 2A par le CIRC, contre 13,6\% (IC95 \% $=13,55 ; 13,58)$ en France pour le même champ $(p=0,001)$. Parmi les salariés exposés, $29,3 \%$, soit 36100 sala- riés, sont exposés à plus de deux produits cancérogènes. Les quatre principaux produits cancérogènes auxquels sont exposés les salariés sont les huiles entières minérales, les gaz d'échappement diesel, les poussières de bois et la silice 
cristalline. Les taux d'exposition régionaux et nationaux à chaque produit cancérogène sont répertoriés dans le tableau I. Dans les Pays de la Loire, les hommes sont cinq fois plus exposés que les femmes aux produits cancérogènes. Près de 110000 hommes (20,6 \% des hommes) sont exposés aux cancérogènes sur leur lieu de travail. Les salariés ligériens, quel que soit leur sexe, sont, de façon statistiquement significative, moins exposés aux produits cancérogènes qu'au niveau national. Cependant, la proportion d'hommes parmi les salariés exposés aux cancérogènes est supérieure, au seuil de $7 \%$, à celle de la France. Les hommes représentent $88,5 \%$ des salariés exposés à des cancérogènes $(85,7 \%$ au niveau national). Les secteurs de la construction, de l'industrie automobile, des industries des biens intermédiaires sont très majoritairement masculins (plus de $75 \%$ d'hommes). Dans ces secteurs, plus d'un quart des salariés est exposé à un produit cancérogène. Plus de $75 \%$ des personnes employées dans l'éducation ainsi que dans la santé-action sociale sont des femmes. Pourtant, dans ces secteurs, les hommes restent les plus exposés, avec une proportion d'exposition respectivement 15 fois et trois fois plus importante que celle des femmes. Ils sont également deux fois plus souvent que les femmes exposés à plusieurs agents cancérogènes $(31,0 \%$ versus $16,6 \%)$.

La catégorie socioprofessionnelle la plus exposée aux cancérogènes est celle des ouvriers, en particulier des ouvriers qualifiés (tableau III). Parmi les salariés exposés aux produits cancérogènes, $81,7 \%$ sont des ouvriers $(69,8 \%$ au niveau national ; $p<0,001)$. Cela représente 100681 ouvriers ligériens. Près des trois quarts de ces ouvriers $(71,0 \%)$ sont des ouvriers qualifiés. Les professions intermédiaires ne représentent que 10,6\% des personnes exposées dans les Pays de la Loire, alors qu'au niveau national elles représentent près de $20 \%$ des salariés exposés $(p<0,001)$. Les secteurs d'activité les plus exposants sont la construction, les industries manufacturières, le commerce et la réparation automobile. 82,7\% des ouvriers masculins exposés, soit 75758 salariés, sont employés dans l'un de ces trois secteurs d'activité.

Notons également que les jeunes de moins de 30 ans sont significativement plus exposés que le reste de la population salariée. $16,6 \%$ des moins de 30 ans sont exposés aux produits cancérogènes, alors que $12,3 \%$ des plus de 30 ans le sont. Au niveau national, 14,9\% des salariés français de moins de 30 ans sont exposés aux produits cancérogènes. Cette différence d'exposition observée entre la France et les Pays de la Loire n'est pas significative. Cependant, la proportion de salariés âgés de moins de 30 ans parmi les salariés exposés aux produits cancérogènes est significativement supérieure dans les Pays de la Loire. Elle représente $32,3 \%$ des salariés exposés dans les Pays de la Loire et $26,2 \%$ au niveau national. Les ligériens âgés de 25 à 29 ans sont deux fois moins employés à des postes de cadres ou professions intellectuelles supérieures que dans le reste de la France. Dans $42 \%$ des cas, ils sont ouvriers (contre $32 \%$ au niveau national).

Concernant le statut de l'emploi, on observe que les apprentis et les intérimaires ont un taux d'exposition supérieur au taux d'exposition régionale (respectivement 20,1 et $15,5 \%$ ), mais cette différence n'est pas statistiquement significative. Les fonctionnaires sont deux fois moins exposés en Pays de la Loire qu'en France (différence significative). Pour les autres statuts, les différences d'exposition entre la France et les Pays de la Loire sont non significatives. $86,2 \%$ des salariés ligériens exposés à un agent cancérogène sont en $\mathrm{CDI}, 5,0 \%$ sont intérimaires, 4,1\% sont apprentis.

\section{Niveaux d'exposition aux cancérogènes}

Les salariés exposés aux cancérogènes sont majoritairement des ouvriers, comme nous l'avons montré précédemment. Nous avons donc choisi de décrire principalement l'exposition de cette population à risque. Les durées d'exposition et les intensités d'exposition aux produits cancérogènes sont données dans le tableau IV. Pour $15,1 \%$ des salariés ligériens exposés, l'intensité d'exposition n'a pas été renseignée. Elle a été mesurée dans 9,4\% des situations d'exposition pour lesquelles elle est renseignée. Elle tient compte des protections collectives existantes.

Des scores d'exposition, croisant l'intensité d'exposition au cancérogène et la durée de l'exposition, ont été construits. Dans un tiers des situations, ce score est élevé (égal à 3, 4 ou 5), indiquant un niveau d'exposition élevé. Près de $86 \%$ de ces

Tableau III

Proportion de salariés ${ }^{a}$ exposés aux produits cancérogènes par catégorie socioprofessionnelle en France et en Pays de la Loire.

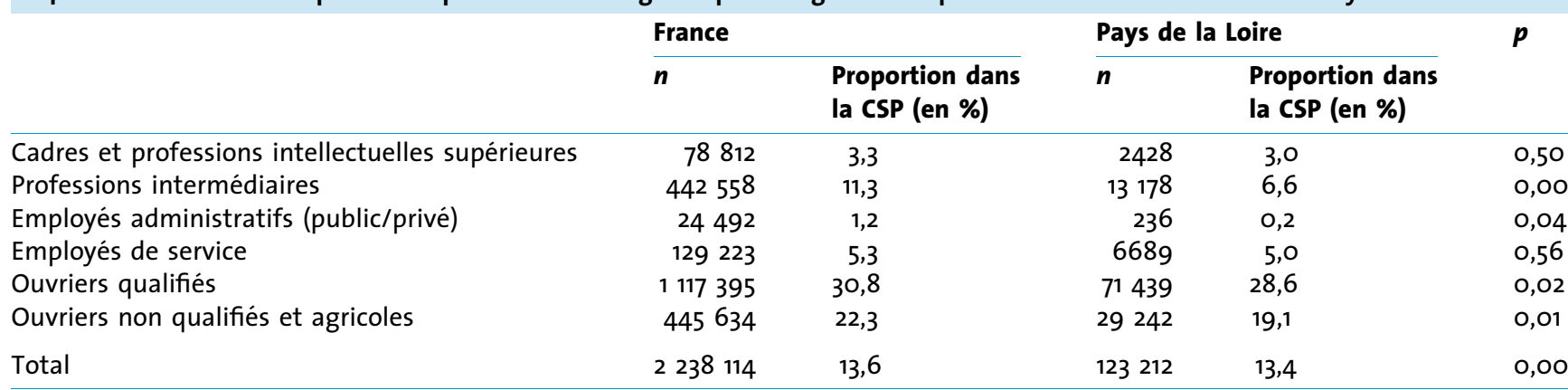

a Salariés du régime général, de la MSA (hors agriculture, pêche, sylviculture), des hôpitaux publics et des intérimaires. 
Tableau IV

Répartition des durées et des intensités hebdomadaires d'exposition aux produits cancérogènes chez les ouvriers.

France

n
Pourcentage

Pourcentage valide

Durée hebdomadaire d'exposition

$<2$ heures

2 à $<10$ heures

10 à $<20$ heures

20 heures ou plus

Données manquantes
94325

656951

211171

507123

34319

$\begin{array}{cc}40,1 & 40,70 \\ 27,9 & 28,30 \\ 9 & 9,10 \\ 21,6 & 21,90 \\ 1,50 & \end{array}$

32,4

38,8

15,1

2,5

11,20

36,50
43,70
17
2,80

Pays de la Loire

n Pourcentage

37,3

$39813 \quad 27,3$

$14456 \quad 9,9$

$32366 \quad 22,2$

4796 $p$

Pourcentage valide

$\begin{array}{ll}38,60 & 0,57 \\ 28,20 & 0,56 \\ 10,20 & 0,44 \\ 22,90 & 0,45\end{array}$

d'expositior

Faible 91306

Forte 355127

Très forte $\quad 59060$

Données manquantes $\quad 263419$

$\begin{array}{rc}36605 & 25,1 \\ 61887 & 42,4 \\ 23212 & 15,9 \\ 2087 & 1,4 \\ 22094 & 15,10\end{array}$

$\begin{array}{ll}29,60 & 0,003 \\ 50 & 0,002 \\ 18,80 & 0,56 \\ 1,70 & 0,09\end{array}$

Salariés du régime général, de la MSA (hors agriculture, pêche, sylviculture), des hôpitaux publics et des intérimaires.

situations concernent les ouvriers. On constate que dans $38,5 \%$ des situations d'exposition, les ouvriers ligériens sont exposés à un niveau élevé. Bien que la différence ne soit pas statistiquement significative, les ouvriers ligériens ont tendance à être exposés à des niveaux plus élevés que les ouvriers français, pour lesquels 35,6 \% des expositions sont de niveau élevé. Si l'on se place à l'échelle du salarié, on ne dénombre pas moins de 28000 ouvriers ligériens exposés à plus de deux produits cancérogènes au cours d'une semaine de travail. Un salarié peut donc être exposé ponctuellement à un produit cancérogène au cours de la semaine et de manière prolongée ou de manière intense à un autre produit cancérogène. Ainsi, 41,9 \% des salariés des Pays de la Loire exposés aux cancérogènes (37,0 \% au niveau national) sont exposés à au moins un produit cancérogène à un niveau élevé (score d'exposition égal à 3, 4 ou 5). Les salariés ligériens, toutes catégories socioprofessionnelles confondues, sont, de manière significative, plus fortement exposés que les salariés français. Les ouvriers sont significativement plus touchés par les expositions élevées que les autres catégories socioprofessionnelles. En effet, la proportion d'ouvriers exposés à au moins un cancérogène de façon élevée parmi les ouvriers ligériens exposés est de $45,5 \%$, ce qui représente 39337 ouvriers. Pour les salariés exposés appartenant aux autres catégories socioprofessionnelles (cadres, professions intermédiaires, employés), le niveau d'exposition est faible dans près de $75 \%$ des cas.

Les industries manufacturières et la construction emploient $73,8 \%$ des salariés fortement exposés. Dans ces deux secteurs, les expositions élevées concernent respectivement 23574 et 9221 salariés, soit $51,4 \%$ et $47,0 \%$ des salariés exposés et employés dans ces secteurs d'activité. Au niveau national, la proportion de salariés fortement exposés est significativement plus faible dans les industries manufacturières (43,2 \%). Dans la construction, 38,7 \% des salariés sont exposés fortement au niveau national. La différence n'est pas significative. Pour les autres secteurs d'activité, l'effectif brut de salariés exposés est faible et ne permet pas d'exploiter les données avec précision. Les travaux de maçonnerie sont les travaux les plus exposants dans le secteur de la construction. Plus d'un quart des salariés exposés dans ce secteur est employé à cette tâche. Dans les industries manufacturières, la métallurgie est l'activité la plus exposante. On dénombre 13549 situations d'exposition dans ce secteur d'activité. Plus de $51 \%$ des salariés de la métallurgie sont exposés à un produit cancérogène. La moitié des salariés travaillant le bois ou fabriquant des objets en bois et des meubles, soit 10573 salariés, est exposée à au moins un produit cancérogène. Plus des trois quarts de ces salariés (7867 salariés) ont des expositions élevées aux cancérogènes. L'effectif brut de salariés exposés dans ces deux secteurs regroupés est de 45 ( 22 dans le travail du bois et la fabrication d'objets en bois, 23 dans la fabrication de meubles). La proportion de salariés exposés de manière élevée dans le secteur de la construction est moins importante (47,0\%). Cependant, dans les secteurs du bois énumérés ci-dessus, une protection collective efficace (aspiration à la source) est présente dans $80,1 \%$ des situations d'exposition élevée. Les moyens mis en place dans le secteur de la construction pour diminuer l'exposition sont beaucoup moins satisfaisants. En effet, une protection collective efficace (aspiration à la source ou vase clos) est présente pour seulement 20,2 \% des situations d'exposition élevée. Dans 64,2 \% des situations d'exposition à niveau élevé, il n'existe donc aucune protection collective. Notons que la protection collective n'a été renseignée que dans $25,1 \%$ des situations d'exposition.

\section{Moyens de prévention mis en place}

Les protections collectives sont mises en place dans un peu plus de la moitié des situations d'exposition chez les ouvriers. Ce chiffre est peut-être sous-estimé. En effet, pour 38,0 \% des expositions, cette donnée n'a pas été renseignée par le médecin enquêteur. Les protections collectives les plus citées 
Tableau V

Exposition aux produits cancérogènes chez les salariés ${ }^{a}$ des Pays de la Loire.

Nom du cancérogène (effectif Répartition des situations d'expositions (en \%) Pourcentage valide de situation d'exposition où il existe une protection collective ${ }^{b}$

Protection individuelle

brut de salariés exposés au

cancérogène)

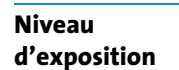

$\begin{array}{ll}\text { Intensité } & \text { Durée } \\ \text { d'exposition } & \text { d'exposition }> \\ \text { forte et très } & 10 \text { heures }\end{array}$

forte et tres

Aucune $^{c}$ Aspiration Vase clos

à la

source

générale

Autre

Protection Pourcentage de

collective données

sans autre manquantes

indication

Produits dont le niveau d'exposition est élevé

Poussières de bois (100)

Nickel et dérivés (31)

54,3

48,6

27,8
15,1
16,2

48,6

Fumées dégagées par les

$65,8 \quad 16,2$

56,1

procédés dans la métallurgie

et l'électrométallurgie (22)

Fumées de vulcanisation (16) $56,2 \quad 7,0 \quad 56,2$

Produits dont le niveau d'exposition est intermediaire

Huiles entières minérales (159) 22,8

Gaz d'échappement diesel (111) 29,8

Silice cristalline (80)

Chrome et dérivés (39) $\quad 36,4$

Goudrons de houille et

dérivés (27)

Cobalt et dérivés (18)

Résines formophénoliques (16)

32,5
43,4
48,5

Carbures métalliques

48,5

frittés (14)

Produits dont le niveau d'exposition est faible

Trichloroéthylène (30)

Formaldéhyde (19)

Amiante (14)

Hydrocarbures aromatiques

17,5
19,8

9,4

7,3

7,3
12,4
28,8

22,5

43,3

17,1

30,4

34,3

35,9
38,8

52,

$\mathbf{5 2 , 1} \quad 0,6$

$\begin{array}{ll}52,1 & 0,6 \\ 30,0 & 0,0\end{array}$

0,0

8,3
22,2

22,2
7,8

$0,0 \quad 3,0$

$\begin{array}{ll}0,0 & 3,0 \\ 0,0 & 9,0\end{array}$

25,8

47

21,6

mise à disposition (en \%)

Protection Protection

respiratoire

halogénés et/ou nitrés (13)

Les pourcentages de protection collective sont en italique lorsque le pourcentage de données manquantes est supérieur à $30 \%$.

a Salariés du régime général, de la MSA (hors agriculture, pêche, sylviculture), des hôpitaux publics et des intérimaires.

${ }^{b}$ Les pourcentages valides sont calculés en ne prenant en compte que les salariés ayant répondu à la question.

¿Le pourcentage de données manquantes est mentionné. Il permet de connaître la proportion exacte de salariés ayant répondu ne pas disposer de protection collective. 
chez les ouvriers sont : l'aspiration à la source $(22,0 \%$ des situations d'exposition), puis la ventilation générale (19,0 \%). Le vase clos est très rarement cité ( $0,7 \%)$. L'existence ou non de protection collective est corrélée, de façon statistiquement significative, au niveau d'exposition des salariés. Ainsi dans plus de la moitié des situations où l'exposition est considérée faible, il n'existe aucune protection collective, alors que dans près de deux tiers des situations d'exposition de niveau élevé, elles sont mises en place. Néanmoins, 13,9\% des ouvriers exposés, soit 14046 ouvriers, sont exposés à un niveau élevé et sans aucune protection collective.

Les équipements de protection individuelle (EPI) respiratoire et/ou cutanée sont mis à disposition des ouvriers dans $45,2 \%$ des cas. Les protections cutanées sont plus souvent présentes aux postes exposant aux cancérogènes que les protections respiratoires (36,9 \% des situations d'exposition contre $15,5 \%$ des situations d'exposition). Dans les situations d'exposition à niveau élevé, les protections respiratoires sont plus souvent mises à disposition que dans les situations où le niveau d'exposition est faible (23,1\% versus $13,6 \%$ chez les ouvriers). Pour les protections cutanées, on n'observe pas de différence significative selon le niveau d'exposition aux cancérogènes. En raison du nombre important de données manquantes concernant la protection collective, nous ne présentons pas les résultats des protections individuelles en fonction des protections collectives.

\section{Les produits cancérogènes}

Le tableau $V$ donne les caractéristiques d'exposition pour chaque produit cancérogène (niveau d'exposition et protections collective et individuelle présentes au poste de travail). Dans l'ensemble des produits cancérogènes considérés, trois groupes émergent quant au niveau des expositions. Le premier groupe comporte quatre produits pour lesquels l'exposition est élevée dans plus de $50 \%$ des cas. Le deuxième groupe est celui pour lequel l'exposition est dite intermédiaire (entre 20 et $50 \%$ des situations d'exposition sont élevées). Enfin, le troisième groupe de produits cancérogènes est celui dont l'exposition est considérée faible (niveau d'exposition bas dans plus de $80 \%$ des situations). Les résultats sont à interpréter avec prudence quand l'effectif brut est inférieur à 30.

\section{Discussion}

Plus de 123000 salariés sont exposés aux produits cancérogènes dans les Pays de la Loire. Les ouvriers, les hommes et les jeunes sont les plus touchés par l'exposition aux cancérogènes. Même si la différence d'exposition des moins de 30 ans dans les Pays de la Loire et la France n'est pas significative, les jeunes ont tendance à être plus exposés dans notre région. Cela peut s'expliquer par le fait que la population salariée ligérienne est plus jeune. En outre, les jeunes occupent dans notre région des postes plus exposants aux cancérogènes.
Certaines expositions sont certainement minorées et d'autres majorées. En effet, la définition retenue pour les produits cancérogènes est celle utilisée par la DARES et l'inspection médicale du travail dans leurs publications sur l'enquête SUMER 2003. Les choix réalisés pour définir les cancérogènes ne sont pas parfaits et sont susceptibles d'être critiqués. Afin d'avoir une vision large des situations d'exposition aux produits cancérogènes, les deux classifications, celle du CIRC et celle de l'UE, ont été retenues. Les classements ne sont pas identiques dans les deux listes. Par exemple, les gaz d'échappement diesel sont 2A pour le CIRC et ne sont pas classés par l'UE. Pour certaines familles, en assimilant certains produits non cancérogènes de cette famille à des produits cancérogènes, les expositions sont majorées. C'est le cas du nickel et de ses dérivés. Le nickel n'est pas cancérogène sous toutes ses formes. Le nickel métal est classé cancérogène $2 \mathrm{~B}$ par le CIRC et les composés du nickel sont classés 1 par le CIRC [12]. Les chaudronniers, les soudeurs inox peuvent être exposés au risque cancérogène du métal durant leur vie professionnelle. Un coiffeur, bien qu'il soit exposé au nickel par l'intermédiaire de ses instruments de coiffure, ne sera pas exposé à ce risque. Plusieurs salariés non exposés à un risque cancérogène ont donc été comptabilisés. Pour d'autres produits cancérogènes, des produits possiblement cancérogènes, voire non classés par le CIRC ont été assimilés aux produits cancérogènes classés 1 ou $2 \mathrm{~A}$ par le CIRC. L'exposition aux goudrons de houille et ses dérivés est ainsi surestimée. Le bitume est pris en compte dans l'effectif de salariés exposés aux goudrons de houille et à ses dérivés. Or, les extraits de bitume sont classés dans le groupe $2 \mathrm{~B}$ du $\mathrm{CIRC}$ et le bitume dans le groupe 3. Le contenu en hydrocarbures polycycliques des bitumes est nettement moins important que celui des goudrons de houille, d'où sa moindre toxicité. Une partie des salariés ayant répondu être exposés aux goudrons de houille et ses dérivés est employée dans le secteur des travaux publics, des transports. Dans les travaux publics, le bitume s'est substitué progressivement aux goudrons les plus toxiques depuis les années 1950 [13]. Les enrobés classiquement utilisés pour la construction des routes sont constitués désormais de bitume mélangé à des agrégats. De plus, il existe actuellement des bitumes antikérosène sans goudrons. Le bitume fluxé, utilisé pour réaliser des enduits superficiels ou des enrobés à froid, est aujourd'hui remplacé le plus souvent par des émulsions de bitumes, appliquées à des températures plus basses [14]. À l'inverse, certaines expositions sont minorées. Le nombre de salariés exposés à l'amiante est probablement sous-estimé par le nombre de salariés exposés aux fibres céramiques réfractaires. Par ailleurs, certains produits cancérogènes ou situations de travail n'ont pas été repérés dans le cadre du questionnaire de cette enquête. Certains produits récemment classés cancérogènes, comme par exemple le plomb et ses dérivés, n'ont pas été retenus car ils n'étaient pas classés en 2003. De plus, le questionnaire SUMER ne repère pas un certain nombre de produits cancérogènes lorsqu'ils sont très 
rares. Néanmoins, les contours de cette description sont probablement très proches de la réalité des expositions aux produits cancérogènes.

Certaines informations apportées par l'enquête SUMER, comme les intensités d'exposition et les dispositifs de protection collective existants, sont susceptibles d'être critiquées. Le nombre important de données manquantes doit, en effet, nous rendre prudents dans l'interprétation des résultats fournis, mais donne une information primordiale sur l'évaluation et la prévention des risques. Pour les intensités d'exposition, l'absence de données dans $15,1 \%$ des situations reflète la difficulté pour le médecin du travail d'apprécier cette caractéristique de l'exposition. Cela peut s'expliquer par le fait qu'il s'agit principalement de produits de dégradation. En effet, dans ce cas, le produit n'est pas introduit délibérément, mais résulte du processus de production et l'intensité de l'exposition est plus difficilement évaluable [7]. La difficulté d'évaluation est d'autant plus grande que le médecin du travail ne dispose pas toujours de données métrologiques récentes permettant d'affirmer que l'intensité d'exposition est inférieure à la valeur limite d'exposition (VLE). Ainsi, seulement 9,4\% des intensités renseignées sont basées sur des mesures réalisées en entreprise. Pour le reste des observations, le médecin du travail a estimé l'intensité de l'exposition. Or, on sait qu'il est difficile d'apprécier la concentration d'un produit chimique dans l'atmosphère. On comprend ainsi que pour certaines expositions, l'intensité ait pu être minorée, que pour d'autres situations, elle ait été majorée, et que pour celles restantes, elle n'ait pas été renseignée. Prenons l'exemple du formaldéhyde. L'analyse des données de la base COLCHIC met en évidence une proportion de $28,1 \%$ de concentrations supérieures à la valeur limite d'exposition professionnelle (VLEP)-huit heures et de $14 \%$ de concentration supérieure à la VLEP-court terme sur les prélèvements d'air effectués en milieu professionnel de 1987 à mai 2005 [15]. Ces chiffres sont bien supérieurs à ceux obtenus à partir des données de l'enquête SUMER $(14,2 \%$ des situations d'exposition ont une intensité supérieure à $50 \%$ de la VLE). Pour les poussières de bois, on fait le même constat. L'exploitation des résultats de mesures d'exposition stockés dans la base de données COLCHIC a permis d'estimer les niveaux d'exposition aux poussières de bois dans les principaux secteurs concernés. Dans le secteur des scieries, $21 \%$ des prélèvements effectués entre 1995 et 2003 étaient entre $0,5 \mathrm{mg} / \mathrm{m}^{3}$ (soit $50 \%$ de la VLEP) et $1 \mathrm{mg} / \mathrm{m}^{3}$, et $35 \%$ dépassaient la VLEP $\left(1 \mathrm{mg} / \mathrm{m}^{3}\right)$. En 2005 , le FCBA (Institut technologique forêt cellulose bois-construction ameublement) a réalisé une campagne d'évaluation de l'exposition aux poussières de bois dans le secteur des scieries. Les résultats sont comparables aux données obtenues à partir de COLCHIC [16]. D'après les données de l'enquête SUMER 2003, l'intensité d'exposition des salariés ligériens exposés à ce cancérogène est forte (plus de $50 \%$ de la VLEP) ou très forte (dépassant la VLEP) dans seulement $27,8 \%$ des cas. Les différences entre nos résultats et les autres sources sont importantes. Le champ des différentes enquêtes n'est pas le même. L'enquête SUMER prend en compte tous les secteurs d'activités exposant au bois. Les niveaux d'exposition varient considérablement selon le secteur industriel et le poste de travail [17]. Certaines professions, comme les couvreurs zingueurs, les charpentiers, sont exposés plus faiblement aux poussières de bois que les ouvriers des scieries. Ces expositions plus faibles expliquent une partie des différences observées entre nos résultats, ceux de la base COLCHIC et ceux obtenus par le FCBA. Notons, cependant, que l'évaluation des niveaux d'exposition et des intensités d'exposition, réalisée, parallèlement à l'enquête SUMER, par les hygiénistes industriels dans l'enquête SUMER hygiène industrielle (HI), est proche de celle réalisée par les médecins enquêteurs de SUMER [18]. Autre écueil de la variable " intensité d'exposition " : elle prend en compte l'existence des protections collectives. Dans une démarche classique d'évaluation des risques, les moyens de prévention des risques ne doivent pas être pris en compte. En les prenant en compte, on sous-estime les dangers. On ne peut pas préjuger des situations d'exposition élevée pour lesquelles une protection collective efficace existe. On ne peut donc pas non plus souligner les efforts réalisés en matière de prévention. Cependant, on approche le risque réel encouru par le salarié et non le risque auquel il aurait été exposé en l'absence de toute protection. Enfin, la définition de l'intensité peut, elle-même, être discutée. Les différents niveaux d'intensité ont été définis à partir des VLEP, qui ont de nombreuses limites. La fixation des VLEP intègre, en effet, non seulement des critères scientifiques et techniques, mais également des critères sociaux et économiques, voire psychologiques [19]. De plus, les VLEP n'intègrent pas la pénibilité de certains travaux (qui modifie la pénétration et donc la toxicité de certains produits) et ne tient pas compte de toutes les voies de pénétration des substances dans l'organisme. Toutes ces limitations reconnues aux VLEP prennent une acuité particulière pour les substances cancérogènes. Dans l'ignorance de l'existence d'un seuil de concentration au-dessous duquel le risque est inexistant, et en raison de l'irréversibilité des effets à craindre, il est primordial de rappeler que les salariés exposés, même à une faible intensité (inférieure à $50 \%$ de la VLEP), ne sont pas protégés du risque cancérogène. Définir des valeurs limites et des niveaux d'exposition est, cependant, nécessaire et fixé par le cadre réglementaire (article L. 4121 du Code du travail). Ainsi, même dans le cas des substances cancérogènes, la prise en compte des VLEP comme élément de référence paraît préférable à l'absence de toute valeur, absence qui peut faire apparaître des situations où le risque ne serait pas évalué et donc potentiellement mal suivi et mal maîtrisé. L'évaluation même approximative de l'intensité d'exposition permet de mettre en avant les situations fortement exposantes. Ainsi, on peut espérer agir dans un premier temps sur ces expositions fortes, sans bien entendu négliger les expositions de faible intensité pour lesquelles le risque cancérogène n'est pas nul. Le score d'exposition connaît bien sûr les mêmes limites que l'intensité d'exposition. Nous avons choisi de définir deux niveaux d'exposition, tout comme l'avaient fait 
la DARES et l'inspection médicale du travail, afin de hiérarchiser les risques. Cela permet de cibler les risques à prévenir en priorité et les secteurs les plus fortement exposés.

La variable concernant la protection collective n'a pas été renseignée pour $38 \%$ des situations d'exposition chez les ouvriers. Ce nombre important de données manquantes met en avant les difficultés du médecin du travail et des salariés eux-mêmes à répondre à la question de la protection collective. Le médecin du travail ne connaît pas tous les postes de travail des milliers de salariés qu'il suit. Le salarié, lui-même, ne sait pas toujours quel moyen de protection collective est mis en place à son poste. Notons que dans le secteur de la construction, les données sur la protection collective manquent pour $40 \%$ des situations d'exposition, alors que dans l'industrie manufacturière, elles manquent dans $27 \%$ des situations. Cette différence entre secteurs d'activité montre que le secteur de la construction, secteur où près de 10 ooo salariés sont exposés à haut niveau, est aussi le secteur pour lequel il est difficile de connaître les moyens de protection mis en œuvre. Cette situation s'explique par la variabilité dans le temps et dans l'espace des situations de travail. Les expositions sont aléatoires, intermittentes, d'intensité et de durée variables dans le bâtiment et travaux publics (BTP) [19]. Cela rend difficile non seulement l'évaluation des risques, mais également la mise en place de moyen de prévention. Toutefois, malgré le nombre important de données manquantes, les résultats globaux obtenus sont concordants avec ce qui a déjà été observé. Ainsi, dans leur campagne de contrôle sur l'utilisation des produits cancérogènes, mutagènes, toxique pour la reproduction (CMR) dans l'industrie, les services déconcentrés du ministère du Travail et les services de prévention des risques professionnels des Caisses régionales d'assurance maladie (CRAM) ont également montré que dans les industries de la mécanique, de la plasturgie, des peintures et des vernis, et dans les secteurs producteurs ou utilisateurs de fibres céramiques réfractaires, il n'existe pas de dispositif de protection collective dans un cas sur deux [20]. Alors que la protection collective constitue la première mesure préventive du risque cancérogène, en cas d'impossibilité technique de substitution du produit CMR, l'enquête SUMER vient renforcer le fait qu'elle est encore trop peu utilisée. De plus, les systèmes de protection collective dits "efficaces " que sont l'aspiration à la source et le vase clos, ne sont mis en place qu'une fois sur cinq et une fois sur 100, respectivement. Rappelons, par ailleurs, que ces derniers devraient faire l'objet d'un contrôle annuel d'efficacité dans le cadre de leur maintenance ce qui n'est pas toujours le cas [21]. Cependant, l'utilisation de systèmes en vase clos est vraisemblablement sous-estimée. Les médecins n'ont, en effet, pas forcément cité les produits concernés, considérant que le salarié n'y avait pas été exposé lors de la dernière semaine travaillée, sauf en cas d'intervention ou de rupture du "vase clos".

Les EPI sont encore trop peu souvent mis à disposition des salariés (45,2 \% des cas chez les ouvriers). Malgré leur mise à disposition, ils ne sont pas toujours utilisés, du fait de la gêne ou de l'inconfort occasionnés lorsqu'ils doivent être portés pendant de longues périodes : poids, chaleur, perte de dextérité... Le port des EPI serait effectif dans près de $80 \%$ des cas pour la protection cutanée et dans près de $70 \%$ des cas pour la protection respiratoire [15]. Bien entendu, les protections individuelles ne doivent être envisagées que lorsque toutes les autres mesures d'élimination ou de réduction des risques s'avèrent insuffisantes ou impossibles à mettre en œuvre. II aurait été intéressant de confronter la mise à disposition des protections individuelles en fonction de l'existence de protections collectives. Cependant, comme nous l'avons souligné précédemment, les protections collectives n'ont pas été renseignées dans près de $40 \%$ des cas, ce qui ne nous permet pas de faire d'analyses plus complètes. Par ailleurs, afin de limiter les risques d'erreur d'interprétation des données obtenues, les effectifs bruts ont été mentionnés, notamment pour la description des situations d'exposition à niveau élevé par secteur d'activité. Ne pouvant pas extrapoler les résultats nationaux, nous avons décidé de présenter les résultats obtenus concernant les protections collectives mises en place dans les secteurs les plus exposés. Malgré la faiblesse des effectifs bruts, la description faite de l'exposition aux cancérogènes et de la prévention technique collective mise en place dans les industries manufacturières et la construction semble refléter plutôt bien la réalité. En effet, dans certains secteurs d'activité comme le travail du bois, la fabrication de meuble, il n'est pas surprenant de voir des expositions élevées aux produits cancérogènes. La prévention collective et individuelle est plus simple à mettre en place dans ces secteurs d'activité. À l'opposé, dans le secteur de la construction, le risque est plus difficilement maîtrisable. Les salariés travaillent parfois sur un site fixe, mais souvent aussi sur différents sites, en extérieur ou en intérieur. Les terrains d'intervention des ouvriers du BTP sont si différents que le risque varie d'un chantier à l'autre. Soulignons aussi que les salariés du BTP sont amenés à travailler avec des salariés d'autres entreprises, et donc à être exposés à un produit cancérogène utilisé par ces autres salariés. On comprend ainsi toute la difficulté qu'il existe à évaluer et à maîtriser non seulement les risques propres à l'activité du salarié, mais aussi les risques liés à l'environnement dans lequel il travaille, environnement variable dans le temps et l'espace. Les résultats obtenus nous semblent cohérents avec la complexité d'évaluation et de prévention des risques observée sur le terrain.

\section{Conclusion}

L'exposition aux cancérogènes dans les Pays de la Loire ressemble dans les grandes lignes à celle observée au niveau national. Nous retrouvons dans notre région les disparités d'exposition entre hommes et femmes, ouvriers et autres catégories socioprofessionnelles, moins de 30 ans et plus de 
30 ans. Cependant, la région des Pays de la Loire reste singulière dans son exposition aux produits cancérogènes. Les ouvriers représentent plus de $80 \%$ des salariés exposés en Pays de la Loire contre $70 \%$ au niveau national. Les expositions concernent, encore plus que dans le reste de la France, les hommes et les moins de 30 ans. Ces différences rendent nécessaire l'exploitation des données propres à notre région. La description nationale de l'exposition nous donne une vision globale des risques auxquels sont exposés les salariés ligériens. Cependant, s'appuyer uniquement sur ces résultats pourrait entraîner une sous-estimation de ces risques. On constate, en effet, qu'en ce qui concerne les niveaux d'exposition, les salariés sont un peu plus fortement exposés dans les Pays de la Loire qu'en France. L'enquête SUMER permet ainsi, malgré ses limites, d'avoir une vision globale de l'exposition des salariés ligériens aux produits cancérogènes et d'estimer le nombre de salariés exposés à ce risque. Elle permet de pointer les déficiences en matière de prévention et d'orienter les actions préventives vers les secteurs les plus à risque que sont la construction, la métallurgie et le commerce et la réparation automobile. L'information et la formation des ouvriers, et en particulier des jeunes, à la prévention des risques sont des priorités dans ces secteurs. Les jeunes sont plus exposés. Les sensibiliser à la prévention est nécessaire si l'on ne veut pas voir apparaître dans plusieurs décennies un grand nombre de pathologies oncologiques. Cette formation doit aussi concerner les apprentis. Les apprentis sont les ouvriers de demain. Les sensibiliser dès le début de leur carrière à la prévention permettra d'instaurer et de pérenniser la culture de la prévention des risques.

\section{Conflit d'intérêt}

Aucun.

\section{Remerciements}

Remerciements à tous les médecins du travail ayant participé à l'enquête SUMER.

\section{Références}

[1] Aouba A, Péquignot F, Le Toullec A, Jougla E. Les causes médicales de décès en France ne 2004 et leur évolution 1980-2004. BEH 2007; no 35-36.
[2] La santé observée dans les Pays de la Loire. ORS. Édition 2007.

[3] Hill C, Doyon F, Sancho-Garnier H. Épidémiologie des cancers. Ed. Flammarion Médecine-Sciences; 1997, 111 p.

[4] Brugère J, Naud C. La reconnaissance des cancers professionnels en Europe. Newsl HESA 2003;27:42-3.

[5] Imbernon E. Estimation du nombre de certains cas de cancers dus à des facteurs professionnels en France. Institut de veille sanitaire; 2003, 28 p.

[6] Cancer : plan de mobilisation nationale 2003-2007. Mission interministérielle pour la lutte contre le cancer; 2003, $44 \mathrm{p}$.

[7] Guignon N, Sandret N. Les expositions aux produits cancérogènes. Prem Synth 2005. $\mathrm{N}^{\circ} 28.1$ (juillet).

[8] Guignon N. Enquête "SUMER 2001-2002 " (surveillance médicale des risques professionnels). Doc Med Travail 2001;86: 161-5.

[9] Arnaudo B, Magaud-Camus I, Sandret N, et al. L'exposition aux risques et aux pénibilités du travail de 1994 à 2003. Premiers résultats de l'enquête SUMER 2003. Prem Synth 2004;52.1:1-8.

[10] SUMER 2003. Guide pour une utilisation régionale des données nationales. DARES. Ministère de l'Emploi, de la Cohésion sociale et du Logement; 2006.

[11] Bilan de l'enquête SUMER 2003 - DARES.

[12] Hoet P. Nickel et composés. EMC Toxicologie-Pathologie professionnelle. 16-004-A-10.

[13] Geraut C. L'essentiel des pathologies professionnelles. Ed. Ellipses marketing.

[14] http://www.atousante.com/risques_professionnels/.

[15] Vincent R, Jeandel B. Exposition professionnelle au formaldéhyde en France : informations fournies par la base de données COLCHIC. INRS. Hygiène et sécurité du travail - Cahiers de notes documentaires ND 2247-203-06.

[16] Poussières de bois - Guide de bonnes pratiques dans le secteur des scieries. ED 6029. INRS; juillet 2008.

[17] Carton M, Goldberg M, Luce D. Exposition professionnelle aux poussières de bois. Effets sur la santé et valeurs limites d'exposition. Rev Epidemiol Sante Publique 2002;50:159-78.

[18] Fevotte J, Bergeret $A$, Guignon $N$, et al. SUMER hygiène industrielle : une enquête en parallèle de SUMER 2003 (TF 171). Doc Med Travail 2008;114:243-65.

[19] Valeurs limites d'exposition professionnelle aux agents chimiques en France. ED 984. INRS; 2006, 20 p.

[20] Boulat JF. Risques professionnels dans le bâtiment et les travaux publics. EMC Pathologie professionnelle et de l'environnement. 16-539-A-10.

[21] Certin JF, Fayol M, Fauquet AL, et al. Résultats de la campagne de contrôle 2006 " inspection du travail - prévention des risques professionnels des CRAM » (avec le soutien technique de I'INRS) sur l'utilisation des agents cancérogènes, mutagènes et toxiques pour la reproduction dans l'industrie. Hygiène et sécurité du travail - Cahiers de notes documentaires PR 27-207. 